\title{
INTRUSION RECOGNITION USING NEURAL NETWORKS
}

\author{
Vladimir Golovko ${ }^{1)}$, Pavel Kochurko ${ }^{2)}$ \\ ${ }^{1)}$ Brest State Technical University, Moskovskaja str. 267, 224017 Brest, Belarus, gva@bstu.by \\ 2) Brest State Technical University, Moskovskaja str. 267, 224017 Brest, Belarus, paulermo@tut.by
}

\begin{abstract}
Intrusion detection techniques are of great importance for computer network protecting because of increasing the number of remote attack using TCP/IP protocols. There exist a number of intrusion detection systems, which are based on different approaches for anomalous behavior detection. This paper focuses on applying neural networks for attack recognition. It is based on multilayer perceptron. The 1999 KDD Cup data set is used for training and testing neural networks. The results of experiments are discussed in the paper.
\end{abstract}

Keywords: Neural networks, intrusion detection systems, network attacks, attack recognition.

\section{INTRODUCTION}

The rapid and extensive growth of Internet technology increases the importance of protecting computer networks from attacks. In the last years the number of network attacks has been raised very promptly that has led to significant problems in different companies. For instance some companies like Yahoo were attacked by DoS (denial of service), costing them millions of dollars.

Intrusion detection systems (IDS) are used as a computer network security tool and permit to alert an administrator in case of attack. The main goal of IDS is to detect and recognize network attacks in real time. Nowadays there exist different approaches for intrusion detection. It is signature analysis, rulebased method, embedded sensors, neural networks, artificial immune systems $[1,2,3,4,5,6]$ and so on. The most of these IDS can detect the known attacks and have poor ability to detect new attacks.

In last years a neural network techniques have been applied and investigated for intrusion detection $[7,8,9,10]$ Such approaches are based on different strategies. So, one of them for anomaly detection use analysis of the audit records, produced by the operating system [8]. The other one is based on network protocol analysis [9].

Among the most wide-spread neural networks are feedforward networks, namely multilayer perceptron (MLP). This network type has been proven to be universal function approximator [11]. Another important feature of MLP is the ability to generalization. Therefore MLP can be powerful tool for design of intrusion detection systems.
This paper presents applying of neural networks for intrusion detection through an examination of network traffic data. It has been shown that denial of service and other network-based attacks are presented in the network traffic data. Therefore using neural networks permits to extract nonlinear relationships between variables from network traffic and to design real-time intrusion detection systems.

We describe the intrusion recognition system, which is based on MLP.

The rest of the paper is organized as follows. The section 2 describes attack classification and training data set. In the section 3 the intrusion detection system is described, based on neural network approach. Section 4 presents experimental results. Conclusion is given in section 5 .

\section{ATTACK CLASSIFICATION AND KDD DATA SET}

An event is a minimal unit with which modern protection tools operate. As soon as event breaks a policy of security, it at once is considered as a part of attack. Action or sequence of the connected actions of the intruder resulting in realization of threat by use of vulnerabilities is called attack to information system.

There are various types of attack classifications. For example, division into passive and active, external and internal attacks, deliberate and unintentional. It should be mentioned that many models of attacks are currently well known: "one-to-one" or "one-to-many", i.e. attack proceeds from one point; "many-to-one" and "many-to-many", i.e. 
distributed or coordinated attacks; hybrid attacks also named the blended threat [12].

In the 1998 DARPA intrusion detection evaluation program, an environment was set up to acquire raw TCP/IP dump data for a network by simulating a typical U.S. Air Force LAN. The LAN was operated like a true environment, but being blasted with multiple attacks [13]. In 1999 sample data set of network traffic was presented at KDD'99 conference [14].

Attacks can be classified on the purposes of intrusion. Some of this categories were used in KDD data set $[12,14]$ :

Remote penetration, R2L - attacks which allow to realize the remote control of a computer through a network: unauthorized access from a remote machine.

Local penetration, U2R - the attack resulting in assigning of non-authorized access to the site on which it is started, unauthorized access to local superuser (root) privileges.

Remote denial of service, DoS - attack which allows to break functioning of system or to overload a computer through Internet.

Local denial of service, DoS - the attack, allowing to break functioning system or to overload a computer on which it is realized. An example of such attack is the hostile applet which loads the central processor an infinite cycle that results in impossibility of transaction processing of other applications.

Scanners, probing - analysis of the topology of a network, services accessible to attack, carrying out search of vulnerabilities on network hosts.

Sniffers - programs which "listen" to the network traffic. Using these programs it is possible to search automatically for identifiers and passwords of users, the information on credit cards, etc.

KDD database consists of 4940210 records where every record describes one TCP/IP connection. Only $20 \%$ of records represent normal connections. A connection is by a sequence of TCP packets during a duration whose starting time and ending time are both well defined, and data flow during this duration from a source IP address to a target IP address under some well defined protocol. Each connection is labeled as either normal or attack. In the latter case, the connection should be with exactly one specific attack type.

For each TCP/IP connection, 41 various quantitative and qualitative features were extracted [14]. This features can be divided into three categories: intrinsic features, i. e., general information related to the connection; traffic features, i. e., statistics related to past connections similar to the current one e. g., number of connections with the same destination host or connections related to the same service in a given time window or within a predefined number of past connections; content features, i. e., features containing information about the data content of packets that could be relevant to discover an intrusion [15]. Each connection record consists of approximately 100 bytes.

\section{SYSTEM DESCRIPTION}

Two approaches to intrusion detection are currently used. The first one, called misuse detection is based on the knowledge of attacker behavior. Intrusion detection system compares current network activity with the known patterns of behaviors of attackers attempting to penetrate the system. The second one, called anomaly detection involves identifying activities that vary from established behavior of users, or groups of users. Anomaly detection though is often highly difficult, as it must be tailored system to system, and sometimes even user to user, as behavior patterns and system usage can vary widely $[12,9,16]$.

Let's examine the block-diagram of the intrusion detection system (Fig. 1). It consists of several stages. At the beginning the system reads traffic data and sends it to the preprocessing module. The task of preprocessing module is to collect necessary data for neural networks from network traffic.

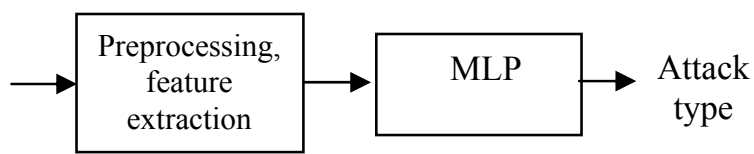

Fig. 1 - Block diagram of the network traftic processing Simplified IDS structure

Our intrusion detection system uses its own sniffer based on WinPCap driver to collect raw traffic data [17]. WinPCap is Windows port of UNIX pcap and is used for sniffing of network devices. It provides gathering data from IP, TCP, UDP, ICMP protocols. Every packet we receive from WinPCap has its header and body. The system analyzes the header data and calculates the parameters of TCP-connections. Every incoming and outgoing packet is analyzed and its parameters are added to the connection parameters. The following set of parameters of TCP-connections (Table 1) are selected by preprocessing module for training and testing of neural networks, like it is shown in Tables 3-5.

Such a system permits to identify and recognize the network attacks.

Let's consider the neural network for recognition of attack. This network is multilayer perceptron with 6 input units, 40 hidden units and 23 output units, where the number of the unit with maximal value 
shows the type of recognized attack (Fig. 2). It should be noted that one MLP for each service has been used. The backpropagation algorithm is used for MLP training.

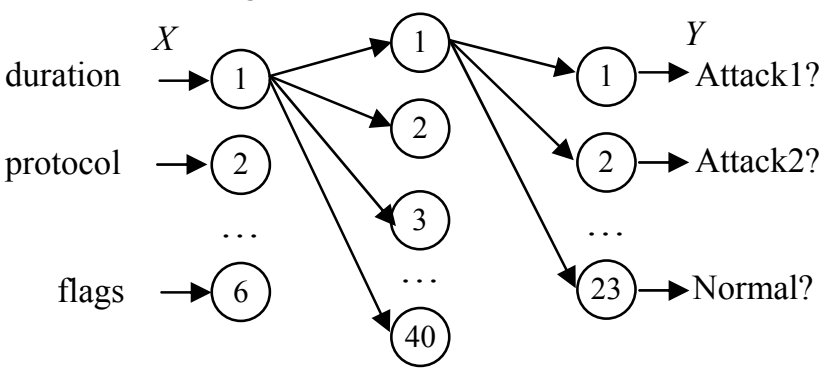

Fig. 2 - MLP structure

The results of experiments are discussed in the next section

\section{EXPERIMENTAL RESULTS}

To assess the effectiveness of the proposed intrusion detection approach, the experiments were conducted on the KDD Cup network intrusion detection data set [14]. Training data sets for recognition of attack consist of normal samples and attacks (Table 2) for each service.

Let's examine the recognition of attack types (Table 3). Table 4 shows the statistic of recognition attacks depending on attack type for some services. Table 5 shows the common results concerning attack recognition and detection for four categories. As can be seen MLP network can recognize 94,49\% attack.

Table 1. Selected network traffic elements

\begin{tabular}{|c|c|c|}
\hline $\begin{array}{l}\text { Feature } \\
\text { name }\end{array}$ & Description & Type \\
\hline duration & $\begin{array}{l}\text { length (number of } \\
\text { seconds) of the } \\
\text { connection }\end{array}$ & cont. \\
\hline protocol type & $\begin{array}{l}\text { type of the protocol, e.g. } \\
\text { tcp, udp, etc. }\end{array}$ & discr. \\
\hline service & $\begin{array}{l}\text { network service on the } \\
\text { destination, e.g., http, } \\
\text { telnet, etc. }\end{array}$ & discr. \\
\hline src bytes & $\begin{array}{l}\text { number of data bytes } \\
\text { from source to } \\
\text { destination }\end{array}$ & cont. \\
\hline dst bytes & $\begin{array}{l}\text { number of data bytes } \\
\text { from destination to } \\
\text { source }\end{array}$ & cont. \\
\hline logged in & $\begin{array}{l}1 \text { if successfully logged } \\
\text { in; } 0 \text { otherwise }\end{array}$ & discr. \\
\hline flags & TCP/IP network flags & discr. \\
\hline
\end{tabular}

Table 2. Training data sets

\begin{tabular}{|l|l|l|}
\hline $\begin{array}{l}\text { Attack } \\
\text { Type }\end{array}$ & $\begin{array}{l}\text { \# of } \\
\text { normal } \\
\text { samples }\end{array}$ & $\begin{array}{l}\text { Total } \\
\text { samples }\end{array}$ \\
\hline auth & 220 & 328 \\
bgp & 0 & 106 \\
domain & 3 & 116 \\
eco i & 109 & 207 \\
finger & 468 & 670 \\
ftp & 190 & 407 \\
ftp data & 350 & 457 \\
http & 219 & 442 \\
pop 3 & 79 & 202 \\
private & 180 & 458 \\
smtp & 79 & 99 \\
telnet & 219 & 513 \\
\hline
\end{tabular}

Table 3. Identification and recognition statistics depending on service

\begin{tabular}{|c|c|c|c|}
\hline Service & $\begin{array}{l}\text { True } \\
\text { Alarms }\end{array}$ & $\begin{array}{l}\text { False } \\
\text { Alarms } \\
\end{array}$ & $\begin{array}{l}\text { Recogn. } \\
\text { Correctly }\end{array}$ \\
\hline 1 auth & $\begin{array}{l}108 \\
100 \% \\
\end{array}$ & 0 & $\begin{array}{l}108 \\
100 \% \\
\end{array}$ \\
\hline 2 bgp & $\begin{array}{l}106 \\
100 \%\end{array}$ & 0 & $\begin{array}{l}0 \\
0 \%\end{array}$ \\
\hline 3 courier & $\begin{array}{l}108 \\
100 \%\end{array}$ & 0 & $\begin{array}{l}88 \\
81,48 \%\end{array}$ \\
\hline 4 csnet ns & $\begin{array}{l}126 \\
100 \%\end{array}$ & 0 & $\begin{array}{l}100 \\
79,37 \%\end{array}$ \\
\hline $5 \mathrm{ctf}$ & $\begin{array}{l}97 \\
100 \%\end{array}$ & 0 & $\begin{array}{l}78 \\
80,41 \%\end{array}$ \\
\hline 6 daytime & $\begin{array}{l}103 \\
100 \%\end{array}$ & 0 & $\begin{array}{l}102 \\
99,03 \%\end{array}$ \\
\hline 7 discard & $\begin{array}{l}116 \\
100 \%\end{array}$ & 0 & $\begin{array}{l}89 \\
76,72 \%\end{array}$ \\
\hline 7 domain & $\begin{array}{l}113 \\
100 \%\end{array}$ & 0 & $\begin{array}{l}112 \\
99,12 \%\end{array}$ \\
\hline 8 domainu & $\begin{array}{l}0 \\
0 \%\end{array}$ & 0 & $\begin{array}{l}0 \\
0 \%\end{array}$ \\
\hline 9 echo & $\begin{array}{l}112 \\
100 \%\end{array}$ & 0 & $\begin{array}{l}89 \\
79,46 \%\end{array}$ \\
\hline 10 eco i & $\begin{array}{l}1253 \\
100 \%\end{array}$ & 0 & $\begin{array}{l}1149 \\
91,7 \%\end{array}$ \\
\hline 11 ecr i & $\begin{array}{l}281049 \\
99,99 \%\end{array}$ & 0 & $\begin{array}{l}280790 \\
99,90 \%\end{array}$ \\
\hline $12 \mathrm{efs}$ & $\begin{array}{l}103 \\
100 \% \\
\end{array}$ & 0 & $\begin{array}{l}79 \\
76,7 \%\end{array}$ \\
\hline 13 exec & $\begin{array}{l}99 \\
100 \%\end{array}$ & 0 & $\begin{array}{l}99 \\
100 \%\end{array}$ \\
\hline 14 finger & $\begin{array}{l}200 \\
99,01 \%\end{array}$ & $\begin{array}{l}3 \\
0,64 \%\end{array}$ & $\begin{array}{l}180 \\
90 \%\end{array}$ \\
\hline $15 \mathrm{ftp}$ & $\begin{array}{l}414 \\
97,41 \%\end{array}$ & $\begin{array}{l}3 \\
0,8 \%\end{array}$ & $\begin{array}{l}409 \\
98,79 \%\end{array}$ \\
\hline
\end{tabular}




\begin{tabular}{|c|c|c|c|c|c|c|c|}
\hline Service & $\begin{array}{l}\text { True } \\
\text { Alarms } \\
\end{array}$ & $\begin{array}{l}\text { False } \\
\text { Alarms }\end{array}$ & $\begin{array}{l}\text { Recogn. } \\
\text { Correctly }\end{array}$ & Service & $\begin{array}{l}\text { True } \\
\text { Alarms }\end{array}$ & $\begin{array}{l}\text { False } \\
\text { Alarms }\end{array}$ & $\begin{array}{l}\text { Recogn. } \\
\text { Correctly }\end{array}$ \\
\hline $\begin{array}{l}18 \text { host- } \\
\text { names }\end{array}$ & $\begin{array}{l}104 \\
100 \% \\
\end{array}$ & 0 & $\begin{array}{l}86 \\
82,69 \% \\
\end{array}$ & $47 \mathrm{ssh}$ & $\begin{array}{l}104 \\
100 \% \\
\end{array}$ & 0 & $\begin{array}{l}102 \\
98,08 \%\end{array}$ \\
\hline 19 http & $\begin{array}{l}2364 \\
98,21 \%\end{array}$ & $\begin{array}{l}220 \\
0,36 \% \\
\end{array}$ & $\begin{array}{l}2362 \\
99,92 \%\end{array}$ & 48 sunrpc & $\begin{array}{l}107 \\
100 \%\end{array}$ & 0 & $\begin{array}{l}86 \\
80,37 \%\end{array}$ \\
\hline 20 http 443 & $\begin{array}{l}99 \\
100 \%\end{array}$ & 0 & $\begin{array}{l}81 \\
81,82 \%\end{array}$ & 49 supdup & $\begin{array}{l}105 \\
100 \%\end{array}$ & 0 & $\begin{array}{l}77 \\
73,33 \%\end{array}$ \\
\hline 21 imap4 & $\begin{array}{l}116 \\
99,15 \%\end{array}$ & 0 & $\begin{array}{l}82 \\
70,69 \%\end{array}$ & 50 systat & $\begin{array}{l}115 \\
100 \%\end{array}$ & 0 & $\begin{array}{l}92 \\
80 \%\end{array}$ \\
\hline 22 irc & $\begin{array}{l}1 \\
100 \%\end{array}$ & $\begin{array}{l}31 \\
73,81 \%\end{array}$ & $\begin{array}{l}1 \\
100 \%\end{array}$ & 51 telnet & $\begin{array}{l}250 \\
85,03 \%\end{array}$ & $\begin{array}{l}3 \\
1,37 \% \\
\end{array}$ & $\begin{array}{l}246 \\
98,4 \% \\
\end{array}$ \\
\hline 23 iso tsap & $\begin{array}{l}115 \\
100 \%\end{array}$ & 0 & $\begin{array}{l}96 \\
83,48 \%\end{array}$ & $52 \mathrm{tftp} \_\mathrm{u}$ & 0 & $\begin{array}{l}1 \\
100 \%\end{array}$ & 0 \\
\hline 24 klogin & $\begin{array}{l}106 \\
100 \%\end{array}$ & 0 & $\begin{array}{l}82 \\
77,36 \%\end{array}$ & 53 time & $\begin{array}{l}103 \\
100 \%\end{array}$ & $\begin{array}{l}2 \\
3,85 \%\end{array}$ & $\begin{array}{l}103 \\
100 \%\end{array}$ \\
\hline 25 kshell & $\begin{array}{l}98 \\
100 \% \\
\end{array}$ & 0 & $\begin{array}{l}82 \\
83,67 \% \\
\end{array}$ & 54 uucp & $\begin{array}{l}106 \\
100 \% \\
\end{array}$ & 0 & $\begin{array}{l}80 \\
75,47 \% \\
\end{array}$ \\
\hline $28 \operatorname{login}$ & $\begin{array}{l}102 \\
98,08 \%\end{array}$ & 0 & $\begin{array}{l}102 \\
100 \%\end{array}$ & 57 whois & $\begin{array}{l}110 \\
100 \%\end{array}$ & 0 & $\begin{array}{l}90 \\
81,82 \%\end{array}$ \\
\hline $29 \mathrm{mtp}$ & $\begin{array}{l}107 \\
100 \%\end{array}$ & 0 & $\begin{array}{l}83 \\
77,57 \%\end{array}$ & $58 \times 11$ & $\begin{array}{l}2 \\
100 \%\end{array}$ & $\begin{array}{l}8 \\
88,89 \%\end{array}$ & $\begin{array}{l}2 \\
100 \%\end{array}$ \\
\hline 30 name & $\begin{array}{l}98 \\
100 \%\end{array}$ & 0 & $\begin{array}{l}78 \\
79,59 \%\end{array}$ & 59 Z39_50 & $\begin{array}{l}92 \\
100 \%\end{array}$ & 0 & $\begin{array}{l}0 \\
0 \%\end{array}$ \\
\hline
\end{tabular}

Table 4. Statistics depending on attack types

\begin{tabular}{|l|l|l|l|} 
dgm & $100 \%$ & & $0 \%$ \\
\hline 32 netbios & 102 & 0 & 82 \\
ns & $100 \%$ & & $80,39 \%$ \\
\hline 33 netbios & 107 & 0 & 0 \\
ssn & $100 \%$ & & $0 \%$ \\
\hline 34 netstat & 95 & 0 & 1 \\
& $100 \%$ & & $1,05 \%$ \\
\hline 35 nnsp & 105 & 0 & 86 \\
& $100 \%$ & & $81,9 \%$ \\
\hline 36 nntp & 108 & 0 & 106 \\
& $100 \%$ & & $98,15 \%$ \\
\hline 37 other & 1602 & 93 & 1228 \\
& $99,81 \%$ & $1,65 \%$ & $76,65 \%$ \\
\hline 38 pop_2 & 101 & 0 & 82 \\
& $100 \%$ & & $81,19 \%$ \\
\hline 39 pop_3 & 122 & 0 & 119 \\
& $99,19 \%$ & & $97,54 \%$ \\
\hline 40 printer & 109 & 0 & 107 \\
& $100 \%$ & & $98,17 \%$ \\
\hline 41 private & 103500 & 2 & 83900 \\
& $99,97 \%$ & $0,03 \%$ & $81,01 \%$ \\
\hline 42 remote & 120 & 0 & 101 \\
job & $100 \%$ & & $84,17 \%$ \\
\hline 43 rje & 111 & 0 & 83 \\
& $100 \%$ & & $74,77 \%$ \\
\hline 44 shell & 111 & 0 & 111 \\
& $100 \%$ & & $100 \%$ \\
\hline 45 smtp & 122 & 28 & 120 \\
& $97,6 \%$ & $0,29 \%$ & $98,36 \%$ \\
\hline 46 sql_net & 110 & 0 & 0 \\
& $100 \%$ & & $0 \%$ \\
\hline
\end{tabular}

\begin{tabular}{|c|c|c|c|}
\hline Attack & Count & Detected & Recogn. \\
\hline 1 back & 2203 & $\begin{array}{l}2192 \\
99,5 \%\end{array}$ & $\begin{array}{l}2192 \\
100 \%\end{array}$ \\
\hline $\begin{array}{l}2 \text { buffer } \\
\text { overflow }\end{array}$ & 30 & $\begin{array}{l}0 \\
0 \%\end{array}$ & $\begin{array}{l}0 \\
0 \%\end{array}$ \\
\hline $3 \mathrm{ftp}$ write & 8 & $\begin{array}{l}2 \\
25 \%\end{array}$ & $\begin{array}{l}2 \\
100 \%\end{array}$ \\
\hline $\begin{array}{l}4 \text { guess } \\
\text { passwd }\end{array}$ & 53 & $\begin{array}{l}49 \\
92,45 \%\end{array}$ & $\begin{array}{l}49 \\
100 \% \\
\end{array}$ \\
\hline 5 imap & 12 & $\begin{array}{l}11 \\
91,67 \% \\
\end{array}$ & $\begin{array}{l}1 \\
9,09 \%\end{array}$ \\
\hline 6 ipsweep & 1247 & $\begin{array}{l}1236 \\
99,12 \%\end{array}$ & $\begin{array}{l}1161 \\
93,93 \%\end{array}$ \\
\hline 7 land & 21 & $\begin{array}{l}21 \\
100 \%\end{array}$ & $\begin{array}{l}0 \\
0 \%\end{array}$ \\
\hline $\begin{array}{l}8 \text { loadmod- } \\
\text { ule }\end{array}$ & 9 & $\begin{array}{l}0 \\
0 \% \\
\end{array}$ & $\begin{array}{l}0 \\
0 \% \\
\end{array}$ \\
\hline 9 multihop & 7 & $\begin{array}{l}1 \\
14,29 \%\end{array}$ & $\begin{array}{l}0 \\
0 \% \\
\end{array}$ \\
\hline 10 neptune & 107201 & $\begin{array}{l}107177 \\
99,98 \%\end{array}$ & $\begin{array}{l}86445 \\
80,6 \%\end{array}$ \\
\hline 11 nmap & 231 & $\begin{array}{l}205 \\
88,74 \%\end{array}$ & $\begin{array}{l}99 \\
48,29 \%\end{array}$ \\
\hline 12 perl & 3 & $\begin{array}{l}0 \\
0 \%\end{array}$ & $\begin{array}{l}0 \\
0 \%\end{array}$ \\
\hline $13 \mathrm{phf}$ & 4 & $\begin{array}{l}2 \\
50 \% \\
\end{array}$ & $\begin{array}{l}2 \\
100 \%\end{array}$ \\
\hline 14 pod & 264 & $\begin{array}{l}259 \\
98,11 \%\end{array}$ & $\begin{array}{l}0 \\
0 \%\end{array}$ \\
\hline
\end{tabular}




\begin{tabular}{|l|l|l|l|}
\hline Attack & Count & Detected & Recogn. \\
\hline $\begin{array}{l}15 \\
\text { portsweep }\end{array}$ & 1040 & 1038 & 498 \\
\hline 16 rootkit & 10 & $\begin{array}{l}99,81 \% \\
20 \%\end{array}$ & $\begin{array}{l}2 \\
10,98 \%\end{array}$ \\
\hline 17 satan & 1589 & $\begin{array}{l}1578 \\
99,31 \%\end{array}$ & $\begin{array}{l}1522 \\
96,45 \%\end{array}$ \\
\hline 18 smurf & 280790 & $\begin{array}{l}280790 \\
100 \%\end{array}$ & $\begin{array}{l}280790 \\
100 \%\end{array}$ \\
\hline 19 spy & 2 & $\begin{array}{l}0 \\
0 \%\end{array}$ & 0 \\
& & 977 \\
\hline 20 teardrop & 979 & 977 \\
& & $99,8 \%$ & $100 \%$ \\
\hline $\begin{array}{l}21 \text { warez- } \\
\text { client }\end{array}$ & 1020 & $\begin{array}{l}427 \\
41,86 \%\end{array}$ & $\begin{array}{l}427 \\
100 \%\end{array}$ \\
\hline $\begin{array}{l}22 \text { warez- } \\
\text { master }\end{array}$ & 20 & $\begin{array}{l}17 \\
85 \%\end{array}$ & $\begin{array}{l}16 \\
94,12 \%\end{array}$ \\
\hline
\end{tabular}

[3]. E. Spafford and D. Zamboni.

Data collection mechanisms for intrusion detection systems, CERIAS Technical Report 2000-08, CERIAS, Pardue University, 1315 Recitation Building, West Lafayette, IN, 2000.

[4]. H. Debar, M. Becke \& D.Simboni. A Neural Network Component for an Intrusion Detection System, In proceedings of the IEEE Computer Society Symposium on Research in Security and Privacy, (1992).

[5]. C. Jirapummin and N. Wattanapongsakorn. Visual Intrusion Detection using SelfOrganizing Maps, Proc. of Electrical and Electronic Conference (EECON-24), Thailand, Vol. 2, pp. 1343-1349, 2001.

[6]. S.C. Lee and D.V. Heinbuch. Training a Neural Network Based Intrusion Detector to Recognize Novel Attacks, Information Assistance and Security, pp. 40-46, 2000.

Table 5. Identification and recognition statistics depending on attack category

\begin{tabular}{|l|l|l|l|}
\hline Category & Count & Detected & Recognized \\
\hline 1 dos & 391458 & 391416 & 370404 \\
& & $99,98 \%$ & $94,62 \%$ \\
\hline 2 u2r & 52 & 2 & 0 \\
& & $3,84 \%$ & $0 \%$ \\
\hline 3 r2l & 1126 & 509 & 497 \\
& & $45,2 \%$ & $97,64 \%$ \\
\hline 4 probe & 4107 & 4057 & 3280 \\
& & $98,78 \%$ & $79,86 \%$ \\
\hline
\end{tabular}

\section{CONCLUSION}

This paper describes the applying of MLP for attack recognition. In comparison with other approaches the neural networks permit to design the intrusion detection systems, which have ability to training and working in real time. The experiments have shown the efficiency of neural networks techniques.

\section{ACKNOWLEDGMENT}

This research is supported by the Grant of Belarus National Academy of sciences.

\section{REFERENCES}

[1]. M. Bishop, S. Cheung, C. Wee, J. Frank, J. Hoagland and S. Samorodin. The treat from the Net, IEEE Spectrum, 34(8), pp. 56-53, 1993.

[2]. D. Anderson, T. Frivold \& A. Valdes. Nextgeneration Intrusion Detection Expert Systems (NIDES): A Summary, SRI International Technical Report SRI-CSL-95-07, 1995.

[7]. S. C. Lee, D. V. Heinbuch, Training a NeuralNetwork Based Intrusion Detector to Recognize Novel Attacks, IEEE Trans. on Systems, Man, and Cybernetics, Part A, 31, 2001, pp. 294-299.

[8]. A. K. Ghosh and A. Schwartzbard, A Study in Using Neural Networks for Anomaly and Misuse Detection, In Proc. of the USENIX Security Symposium, August 23-26, 1999, Washington, USA.

[9]. J. Cannady, An adaptive neural network approach to intrusion detection and response, PhD Thesis, School of Comp. and Inf. Sci., Nova Southeastern University,

[10]. J. M. Bonifacio et al., Neural Networks applied in intrusion detection systems, Proc. of the IEEE World congress on Comp. Intell. (WCCI'98), 1998.

[11]. K. Hornik, M. Stinchcombe, H. White, Multy-layer feedforward networks are universal approximators, Neural Networks, 2 pp. 359-366, 1989.

[12]. A. Lukatsky. Intrusion detection. SaintPetersburg: BHV-Peterburg, 2003.

[13]. MIT Lincoln Laboratory -DARPA Intrusion Detection Evaluation Web Page Template. Training Data Attack Descriptions http://www.ll.mit.edu/IST/ideval/docs/1998/atta $\underline{\text { cks.html }}$

[14]. 1999 KDD Cup Competition. http://kdd.ics.uci.edu/databases/kddcup99/kddcu p99.html

[15]. W. Lee and S. J. Stolfo. A framework for constructing features and models for intrusion detection systems, ACM Trans. on Inform. and System Security, 3(4), 200, 227-261. 
[16]. J. Cannady, Applying Neural Networks to Misuse Detection, In Proceedings of the $21^{\text {st }}$ National Information Systems Security Conference.

[17]. WinPcap: the Free Packet Capture Architecture for Windows, NetGroup, Politecnico di Torino (Italy), http://winpcap.polito.it, 1999-2004.

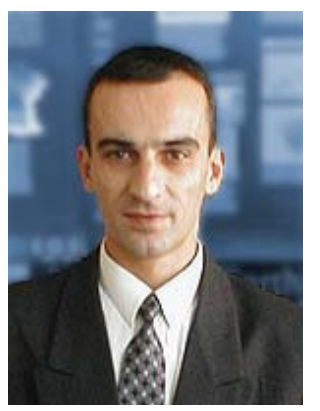

Prof. Vladimir Golovko was born in Belarus in 1960. He received M.E. degree in Computer Engineering in 1984 from the Moscow Bauman State Technical University. In 1990 he received $P h D$ degree from the Belarus State University and in 2003 he received doctor science degree in Computer Science from the United Institute of Informatics problems national Academy of Sciences (Belarus). At present he works as a head of
Intelligence Information Technologies Department and Laboratory of Artificial Neural Networks of the Brest State Technical University. His research interests include Artificial Intelligence, neural networks, autonomous learning robot, signal processing, chaotic processes, intrusion and epilepsy detection. He has published more than 150 scientific papers, including 3 books and 2 chapters of books.

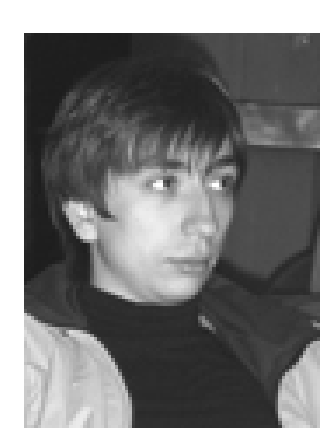

Pavel Kochurko, $2^{\text {nd }}$ year PhD student, Lab. Of Artificial Neural Networks, Dep. Of Intelligent Informational Technologies, Brest State Technical University, Belarus. His research interests include computer network security, data-mining, artificial intelligence and neural networks. In 2002 he has won the prize of "First International Delphic Games of CIS-states" in web-design. 